\title{
Hemorragia incoercible por trauma maxilofacial: experiencia del Hospital del Trabajador*
}

\author{
Drs. PATRICIO ANDRADES C. ${ }^{1}$, JOSÉ LUIS ROMÁN F. ${ }^{1}$, RICARDO BARTEL A. ${ }^{1}$, CLAUDIO BOREL B. ${ }^{1}$, \\ RODRIGO HERNÁNDEZ N. ${ }^{1}$, RENÉ ROJAS S. ${ }^{1}$, JORGE LANKIN B. ${ }^{1}$, RODRIGO VILLALOBOS A. ${ }^{1}$ \\ 1 Servicio de Cirugía Maxilofacial, Hospital del Trabajador. \\ Santiago, Chile.
}

\begin{abstract}
Management of intractable bleeding facial trauma

Introduction: The aim of this paper is to show our experience in the management of intractable bleeding facial trauma during the past 10 years to determine its impact on our environment and outline the basic principles of treatment. Methods: A retrospective study of all patients with maxillofacial trauma and uncontrollable bleeding from 1999 to 2009. Inclusion criteria were oro-nasal bleeding secondary to maxillofacial trauma requiring emergency treatment by a specialist, without other associated lesions that could be a source of bleeding. We obtained demographic information, mechanism of injury, diagnosis of injury, hemodynamic status, type and timing of tamponade, definitive surgical treatment, results and evolution. Results: 21 patients in the study period, a true incidence of $0.002 \%$. 7 patients (33.3\%) with trauma nasoseptal; 5 (23.8\%) with panfacial fractures, and 7 (33.3\%) with variable involvement of the upper and middle face. Nine patients (42.9\%) had isolated facial injuries. Only 5 patients $(23.8 \%)$ had hemodynamic compromise. Thirteen patients (61.9\%) required posterior nasal packing with or without another procedure to control bleeding. Eight patients (38.1\%) required early reduction and internal fixation as definitive treatment. Conclusions: Maxillofacial trauma uncontrollable bleeding is uncommon and rarely is the primary cause of hypovolemic shock. It should be suspected in patients with facial injuries from hours bleeding. The packing, suturing of wounds and reduce/OTS are the mainstays of early treatment.
\end{abstract}

Key words: Facial trauma, intractable bleeding, treatment.

\section{Resumen}

Introducción: El objetivo del presente trabajo es mostrar nuestra experiencia en el manejo de la hemorragia incoercible por trauma facial durante los últimos 10 años, para determinar su incidencia y delinear los principios básicos del tratamiento. Material y Método: Estudio retrospectivo de todos los pacientes con hemorragia incoercible por trauma maxilofacial entre 1999 y 2009. Criterios de inclusión fueron hemorragia oro-nasal secundaria a traumatismo maxilofacial, sin otras lesiones asociadas que pudieran ser fuente de hemorragia, que requirió de tratamiento de urgencia por un especialista. Se obtuvo información demográfica, mecanismo de injuria, diagnóstico del traumatismo, estado hemodinámico, tipo y momento del tratamiento

*Recibido el 7 de noviembre de 2011 y aceptado para publicación el 12 de diciembre de 2011.

Correspondencia: Dr. Patricio Andrades C.

Av. Vicuña Mackena 200, Santiago, Chile.

pandrades@u.uchile.cl 
especializado, tratamiento quirúrgico definitivo, resultados y evolución. Resultados: 21 pacientes en el período de estudio, con incidencia real de 0,002\%. Destacan 7 pacientes (33,3\%) con trauma nasoseptal, con o sin heridas faciales; 5 (23,8\%) con fracturas panfaciales; 7 (33,3\%) con compromiso variable del tercio superior y medio de la cara. Nueve pacientes (42,9\%) presentaron lesiones faciales aisladas. 5 pacientes (23,8\%) presentaron compromiso hemodinámico. Trece pacientes (61,9\%) necesitaron un taponamiento nasal posterior, asociado o no a otro procedimiento para el control de la hemorragia. Ocho pacientes $(38,1 \%)$ necesitaron reducción y osteosíntesis precoz como tratamiento definitivo. Conclusiones: La hemorragia incoercible por trauma maxilofacial es poco frecuente y rara vez es la causa primaria de shock hipovolémico. Se la debe sospechar especialmente en pacientes con lesiones faciales que llevan horas con sangrado, aún de poca cuantía, pero persistente. El taponamiento, sutura de heridas y reducción/OTS precoz son los pilares de su tratamiento.

Palabras clave: Trauma facial, hemorragia incoercible, tratamiento.

\section{Introducción}

La hemorragia secundaria a un trauma facial es por lo general de poca o mediana cuantía, ocasionalmente puede ser de difícil control por originarse en estructuras más profundas, y a veces puede dificultar la evaluación y el manejo inicial del paciente. En raras ocasiones la hemorragia por trauma facial puede ser masiva y causar shock hipovolémico con riesgo vital. Sin embargo, recientes estudios afirman que esto no es tan infrecuente y que su manejo debiera incluirse obligatoriamente en el curso ATLS (Advanced Trauma Life Support) ${ }^{1}$. Incluso, recomiendan complicados algoritmos de manejo que incluyen procedimientos que no siempre están disponibles para el tratamiento agudo de estos enfermos ${ }^{2}$.

La incidencia de hemorragia masiva con riesgo vital puede llegar hasta un $10 \%$ en pacientes con trauma facial severo ${ }^{3}$. Sin embargo, si se consideran todos los tipos de trauma facial, esta incidencia es mucho menor. Dean et $\mathrm{al}^{2}$, reportan su experiencia de 20 años en hemorragia masiva secundaria a trauma facial, y determinaron una incidencia de $0,33 \%$. Ardekian et $\mathrm{al}^{4}$, muestran una gran variabilidad en la incidencia reportada de esta patología que va desde el 0 al $11 \%$ de los traumas faciales. Bynoe et $\mathrm{al}^{5}$, encuentran que la real incidencia de hemorragia exanguinante en trauma de cara es de 1,2\% en 912 casos. Lamentablemente, el carácter retrospectivo de los estudios, la ausencia de una definición clara de hemorragia masiva por trauma facial, y la dificultad clínica para poder diferenciar claramente si la hemorragia masiva es secundaria al trauma facial o a otro trauma (abdominal, toráxico, extremidades) en pacientes politraumatizados, hace muy dificultoso el cálculo real de la incidencia de este fenómeno.

El objetivo del presente trabajo es mostrar la experiencia del Equipo de Cirugía Maxilofacial del Hospital del Trabajador de Santiago en el manejo de la hemorragia incoercible por trauma facial. Se revisaron los resultados de los últimos 10 años para intentar determinar su incidencia en nuestro medio y delinear los principios básicos del tratamiento.

\section{Pacientes y Método}

\section{Criterios de inclusión}

Se recolectaron datos retrospectivos, obtenidos de fichas clínicas de pacientes atendidos en el Servicio de Cirugía Maxilofacial del Hospital del Trabajador de Santiago, entre los años 1999 a 2009. Los criterios de inclusión fueron hemorragia oro-nasal secundaria a traumatismo maxilofacial que requirió de tratamiento de urgencia por el especialista, sin otras lesiones asociadas que pudieran ser fuente de hemorragia. Utilizando la base de datos computacional del hospital se buscaron los pacientes con los siguientes diagnósticos de alta: fractura malar, maxilar, nasal, orbitaria, base de cráneo y mandibular. El código de estos diagnósticos fue cruzado con códigos relacionados a epistaxis o algún procedimiento para controlar la hemorragia, taponamiento nasal o transfusión sanguínea.

\section{Manejo de la hemorragia por trauma maxilofacial}

En nuestro hospital, siempre que existe un paciente con una hemorragia facial activa y significativa, secundaria a un trauma maxilofacial, el especialista de turno es llamado para resolver el problema. Nosotros definimos una hemorragia facial activa y significativa secundaria a un trauma maxilofacial, a todo sangramiento evidente por cavidades nasoorales, heridas faciales u otro orificio facial que no cede con la compresión y el taponamiento nasal inicial en el servicio de urgencia. En todos estos casos se asegura una vía aérea permeable y se obtiene una vía venosa periférica, para la reposición de volumen y eventual transfusión sanguínea. Ante la imposibilidad de controlar el sangrado con el taponamiento anterior, el paciente es estabilizado hemodinámicamente y llevado a pabellón, donde bajo anestesia general, adecuada iluminación y aspiración central, 
se procede a realizar el control de la hemorragia a través de sutura de heridas y taponamiento. Después de detenida la hemorragia, se procede a la reducción y osteosíntesis de las fracturas faciales en forma precoz (días después del taponamiento). La reducción y contención de las fracturas puede ser también una medida adicional de control de la hemorragia y solución definitiva del problema.

Una de las maniobras básicas para controlar una hemorragia facial es el taponamiento nasal posterior. Los materiales esenciales para realizar un taponamiento nasal posterior clásico son: gasas y algodón, lino 2/0 largo, rinoscopio (espéculo nasal), pinza bayoneta, cánula de aspiración y baja lengua. El primer paso es preparar el tapón de gasa que se va a colocar en la rinofaringe. Esto se realiza con una gasa larga enrollada a la cual se le amarran 2 hilos largos en un sentido y 1 hilo más corto central en otro sentido (Figura 1). Seguidamente se introduce una sonda Foley por cada fosa nasal que se extraen por boca. $\mathrm{Al}$ extremo de cada una se amarran los 2 hilos largos de la gasa tapón. Las sondas se retiran, los hilos se recogen por la nariz y se traccionan para dirigir el tapón a la rinofaringe. Una vez ahí, y manteniendo la tracción de los hilos, se procede a realizar un taponamiento nasal anterior con gasas parafinadas. A continuación, se introduce una tórula de algodón que ocupa el volumen del vestíbulo nasal, los hilos se amarran traccionando el tapón faríngeo, sobre ese algodón. De este modo el taponaje anterior queda comprimido entre el tapón faríngeo y la tórula del vestíbulo nasal para evitar dañar la columela de la nariz. El hilo central se extrae por boca y se deja fijo a la mejilla con tela adhesiva para retirar el taponamiento en 3 a 5 días (Figura 2).

\section{Análisis de datos}

Los datos obtenidos fueron tabulados en una planilla Excel para su análisis e incluyeron información demográfica, mecanismo de injuria, diagnóstico del traumatismo, estado hemodinámico, tipo y momento del taponamiento, tratamiento quirúrgico definitivo, resultados y evolución. El cálculo de prevalencia se realizó dividiendo el número de casos encontrados por el total de consultas durante los años de estudio. La media y la desviación estándar se utilizaron para describir las variables continuas y las frecuencias se utilizaron para resumir las variables categóricas.

\section{Resultados}

Entre los años 1999 y 2009 se realizaron 60.826 consultas y 4.174 cirugías en el Servicio de Cirugía Maxilofacial del Hospital del Trabajador de Santiago. De las consultas, 10.455 (17,2\%) fueron por pacientes nuevos, $45.551(74,9 \%)$ por controles y
4.820 (7,9\%) por procedimientos ambulatorios realizados en la consulta. En este trabajo, un total de 21 pacientes cumplieron los criterios de inclusión, es decir, presentaron una hemorragia incoercible por trauma maxilofacial que requirió de un tratamiento de urgencia para controlar el sangrado, sin otra fuente hemorrágica. No se encontraron pacientes politraumatizados con varias fuentes de sangramiento en los cuales el componente maxilofacial fuera de importancia y requiriera algún tipo de procedimiento inmediato para el control de la hemorragia. De esta forma, si consideramos el total de consultas, la prevalencia de hemorragia por trauma maxilofacial en este estudio es de $0,00036 \%$, pero siendo más precisos, si consideramos el total de consultas por pacientes nuevos como denominador, la prevalencia real es de $0,002 \%$.

Los datos demográficos y clínicos de los 21 pacientes estudiados se resumen en la Tabla 1 . Se encontraron 19 hombres (90\%) y 2 mujeres (10\%) con una edad promedio de 43,7 $\pm 16,49$ años (rango 24-78). Los mecanismos del trauma fueron: $7(33,3 \%)$ por golpe con objeto contundente; 6 (28,6\%) por accidentes de tránsito; 4 (19,1\%) por caída de altura; 2 (9,5\%) por asalto; y 2 (9,5\%) por complicaciones postoperatorias de un trauma maxilofacial. Dentro de los tipos de lesiones destacan: 7 pacientes $(33,3 \%)$ con trauma nasoseptal con o sin heridas faciales, pero sin otras lesiones en el macizo maxilofacial; 5 (23,8\%) con fracturas panfaciales; y 7 (33,3\%) con compromiso variable del tercio superior y medio de la cara. Nueve pacientes (42,9\%) presentaron sólo lesiones en la cara, 7 (33,3\%) presentaron además un TEC abierto, $2(9,5 \%)$ un TEC cerrado, 2 (9,5\%) lesiones menores en extremidades, y $1(4,8 \%)$ una lesión en columna cervical. Sólo 5 pacientes $(23,8 \%)$ presentaron compromiso hemodinámico.

Desde el punto de vista terapéutico, 3 pacientes $(14,3 \%)$ con TEC abierto respondieron adecuadamente al taponamiento nasal anterior. Cinco pacientes $(23,8 \%)$ con fracturas nasoseptales y heridas faciales requirieron una reducción nasoseptal, sutura de heridas y taponamiento anterior como tratamiento de urgencia y definitivo de sus lesiones. El resto de los pacientes, 13 (61,9\%) necesitaron un taponamiento nasal posterior asociado o no a otro procedimiento para el control de la hemorragia. Del total de pacientes, $12(57,1 \%)$ no requirieron tratamiento definitivo; 8 pacientes $(38,1 \%)$ necesitaron reducción y osteosíntesis un promedio de 10,75 \pm 13,32 (rango 2-40) días después del accidente. Sólo 2 pacientes $(9,5 \%)$ no tuvieron una evolución favorable, el primero quedó con severas secuelas neurológicas y el segundo fallece secundario a su TEC grave y a una falla respiratoria. 


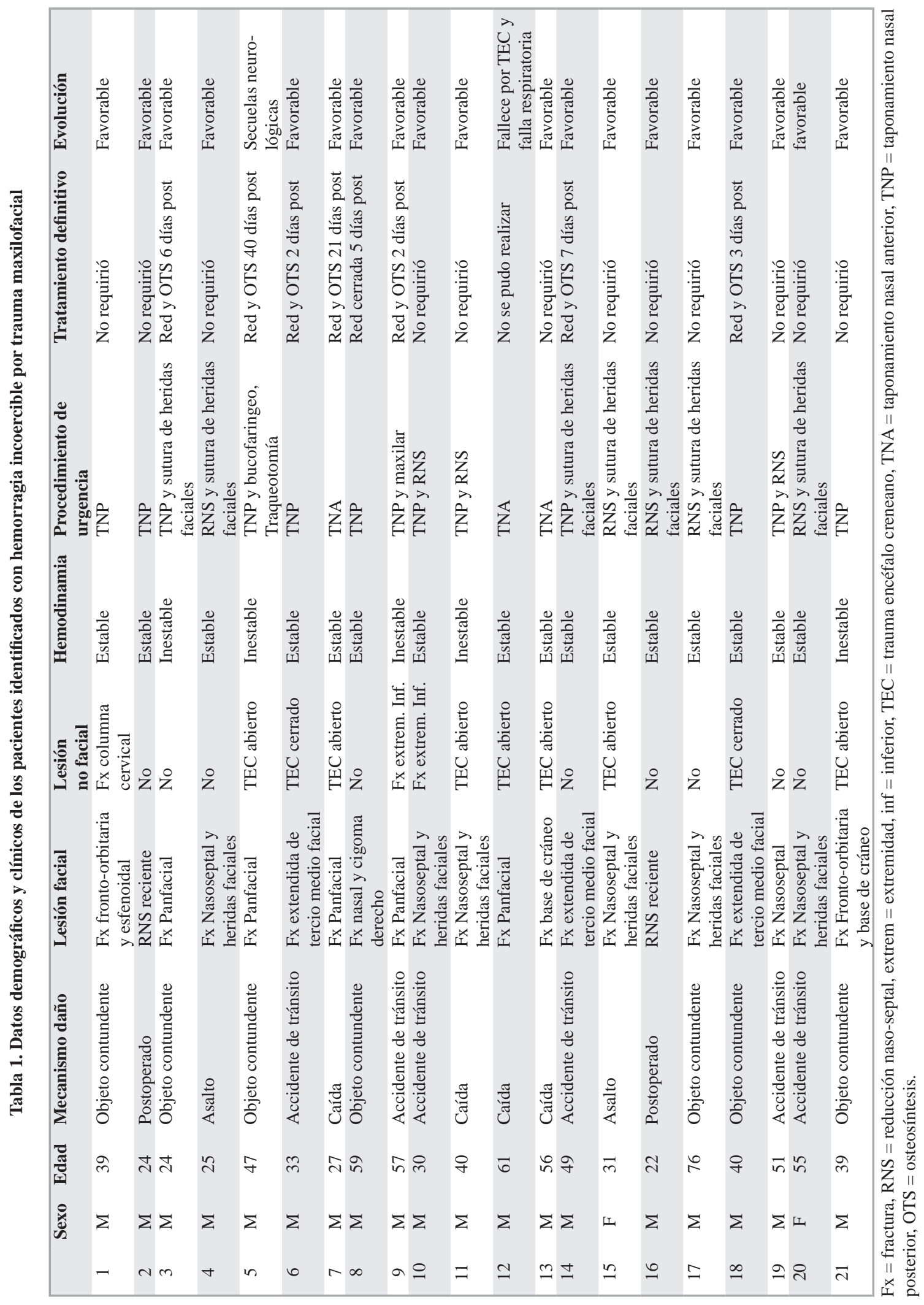



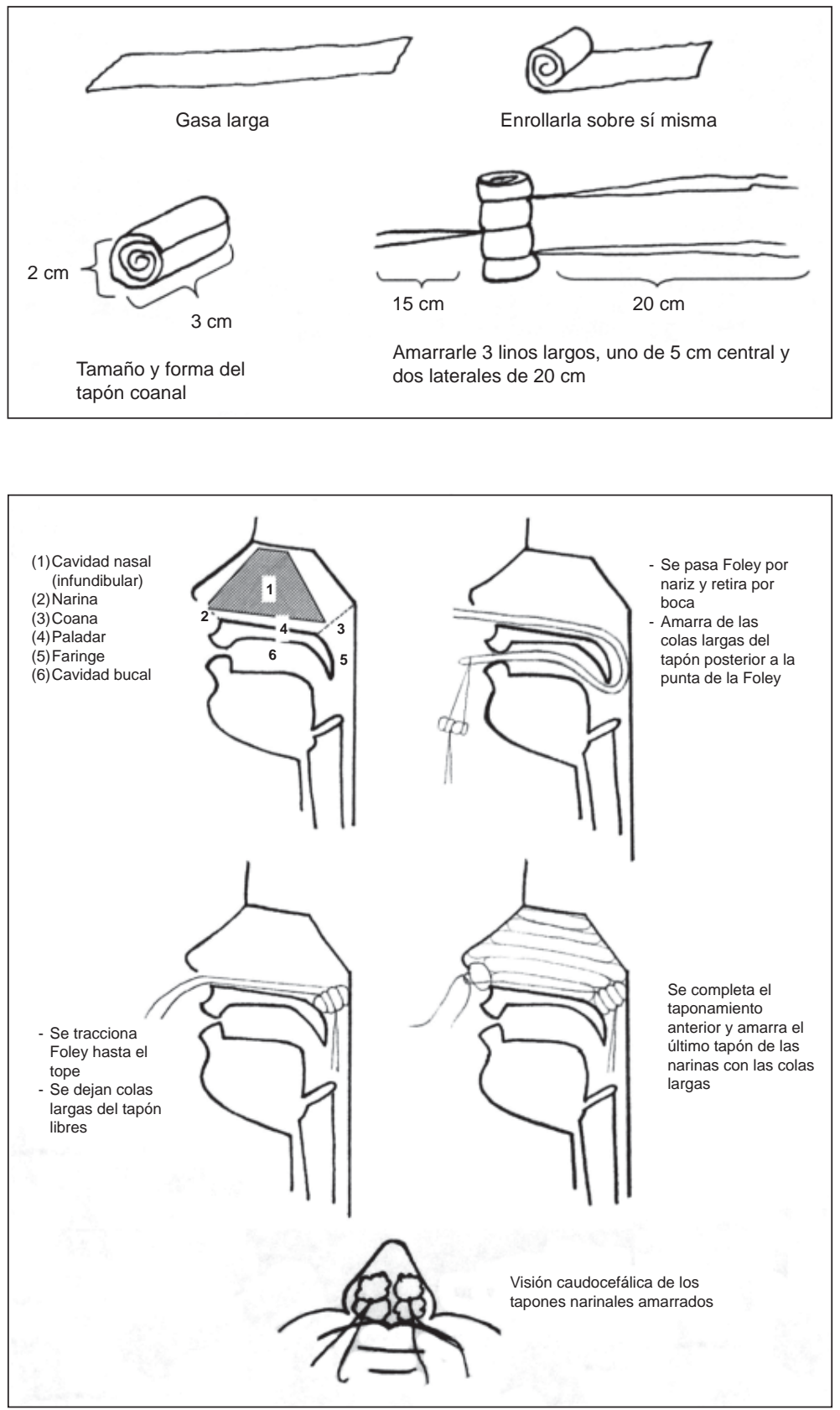

Figura 1. Esquema de confección del tapón posterior o coanal.
Figura 2. Esquema de taponamiento nasal posterior.

\section{Discusión}

La hemorragia por trauma facial es una entidad subvalorada pero en nuestra experiencia, es muy raro que sea potencialmente letal. En los 10 años de nuestro estudio, no se identificó ningún caso de muerte atribuible en mayor o menor medida a una hemorragia por traumatismo maxilofacial. Así como lo reporta Dean et al en su estudio², todos los pacien- tes que presentaron sangramiento por trauma facial sin otras lesiones sobrevivieron, en cambio, la mitad de los pacientes con trauma facial asociado a otro trauma (politraumatizado) fallecieron por causas no siempre relacionadas a la hemorragia facial. Por otro lado, el colegio americano de cirujanos define hemorragia masiva (tipo III) como toda aquella en la que se ha perdido entre un 30 a un $40 \%$ del volumen sanguíneo, lo que se traduce en hipotensión, 
taquicardia sobre 140, taquipnea, oliguria, ansiedad y confusión ${ }^{6}$. Para que una hemorragia así ocurra en forma aguda tiene que existir una lesión grave de grandes vasos y órganos altamente perfundidos. En la cara existe una excelente irrigación, pero la mayoría de los vasos son de pequeño calibre y relativo bajo flujo. Por esta razón, aunque la pérdida de sangre del cuero cabelludo, cara y cuello puede ser evidente, rara vez es rápidamente abundante. El problema es que no se le da la atención necesaria en la evaluación primaria y secundaria. Además, el sangrado del cuero cabelludo puede pasar inadvertido con el paciente en posición supina inmovilizado y el sangramiento de tercio facial medio e inferior, pueden ser deglutidos y pasar a la vía digestiva. Esta falta de atención y demostración clínica puede hacer que la hemorragia proveniente de estas zonas sea relevante no en forma inmediata, pero sí después de algunas horas. De esta manera, fracturas faciales como de huesos propios nasales y mandibulares simples, pueden sangrar de a poco durante algunas horas, causar compromiso hemodinámico diferido y requerir resucitación agresiva.

La hemorragia facial significativa es relativamente poco común, y eso se refleja en los resultados de nuestro estudio y en la literatura. En este trabajo se observó una prevalencia de 2 pacientes con hemorragia facial significativa por cada 1.000 atenciones nuevas. Esta baja prevalencia se debe en parte a que, en el contexto del paciente politraumatizado y hemodinámicamente comprometido, es extremadamente difícil determinar el rol que juega la hemorragia facial en la generación del shock. Sin duda que contribuye, pero el trauma abdominal, torácico, de huesos largos y encefálico juegan un rol más preponderante ${ }^{1}$. Dicho de otra forma, cuando existe shock hipovolémico en el contexto de un paciente politraumatizado, es muy poco probable que las lesiones faciales sean la única causa del shock. Por ese motivo, en este estudio se incluyeron solamente hemorragias incoercibles en el servicio de urgencia, sin otro traumatismo importante que pudiera ser causa de hipovolemia. Además se incluyeron todos los pacientes en los cuales el especialista tuvo que acudir al servicio de urgencia para el manejo inicial, y no sólo los pacientes hemodinámicamente inestables. Concordante con la literatura, pudimos observar que lesiones del tercio medio facial o mayores, sangran con mayor rapidez y pueden convertirse prontamente en significativas. Sólo 5 pacientes se presentaron con shock hipovolémico secundario a hemorragia por trauma maxilofacial aislado: 3 con fractura panfacial y 2 con fracturas extendidas de tercio medio. En estos pacientes la pérdida de sangre ocurre en varios sitios a lo largo de la fractura y de los tejidos blandos asociados, en lugar de un solo vaso sanguíneo, lo que hace que la hemorragia sea abundante y de difícil control.

Todos estos enfermos deben ser manejados bajo las directrices del ATLS ${ }^{6}$. La vía aérea y la protección de la columna cervical son el primer paso a seguir. La intubación y sedación del paciente puede complicar el manejo debido a los efectos de medicamentos en la presión sanguínea y actividad cerebral. Además la inflamación de partes blandas en el cuello puede hacer muy necesaria la intubación para una adecuada ventilación ${ }^{7,8}$. Y en el punto referente a la circulación, se debe realizar una evaluación cuidadosa, vigilando el sangrado de otros lugares y cualquier otra causa de shock no hipovolémico. En la literatura se describen varios enfoques para el control de la hemorragia en estos pacientes. Algunos grupos utilizaron catéteres con balón y reducción con alambres ${ }^{1,9-11}$. Otros no usaron fijación intermaxilar o métodos de OTS en el tratamiento agudo de las fracturas sino que difieren el tratamiento todo lo que sea necesario ${ }^{2}$. Lynham et $\mathrm{al}^{12}$ y Gwyn et $\mathrm{al}^{13}$, utilizan reducción, fijación con alambres y taponamiento nasal con catéter así como la ligadura vascular. Y estudios recientes recomiendan la embolización precoz para el control de la hemorragia facial $^{10,11}$. En nuestra opinión el taponamiento con sonda Foley u otro tipo de balón nasal es poco controlado y tiene el riesgo de producir isquemia en mucosas y piel con la consiguiente necrosis y formación de escaras. Y la embolización selectiva suele ser ineficaz para controlar múltiples focos de sangrado en el territorio facial ricamente irrigado, requiere tener un equipo de alto costo, disponible siempre, muy rápido y eficaz en la realización de procedimientos en pacientes hipovolémicos, y que considere las posibles complicaciones graves secundarias a la embolización, como la isquemia de tejidos no deseados.

Habiendo hecho la evaluación primaria y secundaria, y con el paciente estable, nosotros creemos que un paciente con hemorragia incoercible por trauma maxilofacial debe ser tratado en pabellón bajo anestesia general. Proponemos como principios básicos del tratamiento quirúrgico en estos enfermos: 1) taponamiento adecuado de cavidades; 2) sutura de todas las lesiones de partes blandas, y 3) tratamiento precoz de las fracturas faciales. En nuestro estudio, 8 pacientes respondieron al taponamiento nasal anterior, y 13 requirieron taponamiento posterior, de los cuales 2 además requirieron taponamiento maxilar y bucofaríngeo, logrando en todos, el adecuado control de la hemorragia. A excepción de 2 pacientes graves, todos los pacientes que requirieron reducción y osteosíntesis, se les realizó a pocos días del accidente, como medida adicional del control de la hemorragia. Todo lo anterior se debe realizar en 
pabellón, donde se puede actuar más cómodo, con el paciente bajo anestesia general, y de esta forma poder realizar todas la maniobras necesarias para el adecuado control de la hemorragia. Esta propuesta de acción es práctica y sencilla, puede ser llevada a cabo por un cirujano general, en un hospital donde al menos cuente con pabellón y anestesista de turno, logrando de esta manera una estabilización adecuada para el traslado a un centro de mayor complejidad.

\section{Referencias}

1. Perry M. Advanced Trauma Life Support (ATLS) and facial trauma: can one size fit all? Part 1: dilemmas in the management of the multiply injured patient with coexisting facial injuries. Int J Oral Maxillofac Surg. 2008:37:209-14.

2. Dean NR, Ledgard JP, Katsaros J. Massive hemorrhage in facial fracture patients: definition, incidence, and management. Plast Reconstr Surg. 2009:123:680-90.

3. Perry M, O’Hare J, Porter G. Advanced Trauma Life Support (ATLS) and facial trauma: can one size fit all? Part 3: Hypovolaemia and facial injuries in the multiply injured patient. Int J Oral Maxillofac Sur 2008:37:40514.

4. Ardekian L, Rosen D, Klein Y, Peled M, Michelson M, Laufer D. Life-threatening complications and irreversible damage following maxillofacial trauma. Injury 1998:29:253-6.

5. Bynoe RP, Kerwin AJ, Parker HH, 3rd, Nottingham JM, Bell RM, Yost MJ, et al. Maxillofacial injuries and lifethreatening hemorrhage: treatment with transcatheter arterial embolization. J Trauma 2003:55:74-9.

6. American College of Surgeons. Advanced Trauma Life Support Course for Doctors. 8th ed. Chicago: American College of Surgeons; 2008.
7. Perry M, Morris C. Advanced trauma life support (ATLS) and facial trauma: can one size fit all? Part 2: ATLS, maxillofacial injuries and airway management dilemmas. Int J Oral Maxillofac Surg. 2008;37:309-20.

8. Perry M, Moutray T. Advanced Trauma Life Support (ATLS) and facial trauma: can one size fit all? Part 4: 'can the patient see?' Timely diagnosis, dilemmas and pitfalls in the multiply injured, poorly responsive/unresponsive patient. Int J Oral Maxillofac Surg. 2008;37:505-14.

9. Cannell H, Silvester KC, O’Regan MB. Early management of multiply injured patients with maxillofacial injuries transferred to hospital by helicopter. Br J Oral Maxillofac Surg. 1993;31:207-12.

10. Perry M, Dancey A, Mireskandari K, Oakley P, Davies S, Cameron M. Emergency care in facial trauma: A maxillofacial and ophthalmic perspective. Injury Int $\mathrm{J}$ Care Injured 2005;36:875-96.

11. Tung TC, Tseng WS, Chen CT, Lai JP, Chen YR. Acute life-threatening injuries in facial fracture patients: A review of 1,025 patients. J Trauma Injury Infection Crit Care 2000;49:420-4.

12. Lynham AJ, Hirst JP, Cosson JA, Chapman PJ, McEniery P. Emergency department management of maxillofacial trauma. Emerg Med Australasia 2004;16:7-12.

13. Gwyn PP, Carraway JH, Horton CE, Adamson JE, Mladick RA. Facial fractures: Associated injuries and complications. Plast Reconstr Surg. 1971;47:225-30.

14. Yang WG, Tsai TR, Hung CC, Tung TC. Life-threatening bleeding in a facial fracture. Ann Plast Surg. 2001;46:159-62

15. Bynoe RP, Kerwin AJ, Parker HH, Nottingham JM, Bell RM, Yost MJ, et al. Maxillofacial injuries and lifethreatening hemorrhage: Treatment with transcatheterarterial embolization. J Trauma Injury Infection Crit Care 2003;55:74-9. 